\title{
Solving the standard model problems in softened gravity
}

\author{
Alberto Salvio \\ CERN, Theory Division, CH-1211 Geneva 23, Switzerland \\ (Received 5 August 2016; published 16 November 2016)
}

\begin{abstract}
The Higgs naturalness problem is solved if the growth of Einstein's gravitational interaction is softened at an energy $\lesssim 10^{11} \mathrm{GeV}$ (softened gravity). We work here within an explicit realization where the EinsteinHilbert Lagrangian is extended to include terms quadratic in the curvature and a nonminimal coupling with the Higgs. We show that this solution is preserved by adding three right-handed neutrinos with masses below the electroweak scale, accounting for neutrino oscillations, dark matter and the baryon asymmetry. The smallness of the right-handed neutrino masses (compared to the Planck scale) and the QCD $\theta$-term are also shown to be natural. We prove that a possible gravitational source of $C P$ violation cannot spoil the model, thanks to the presence of right-handed neutrinos. Inflation is approximately described by the Starobinsky model in this context and can occur even if we live in a metastable vacuum.
\end{abstract}

DOI: 10.1103/PhysRevD.94.096007

\section{INTRODUCTION AND SUMMARY}

The hierarchy problem consists in finding an extension of the Standard Model (SM) where the Higgs mass $M_{h}$ is natural: quantum corrections to $M_{h}$ are small compared to its observed value. A challenge is to achieve this in the presence of gravity. Softened gravity is a scenario in which the growth of Einstein's gravitational interaction stops at a scale no larger than $10^{11} \mathrm{GeV}$ [1]. In such a situation, the gravitational quantum corrections to $M_{h}$ are not too large solving the hierarchy problem. An important question is whether this scenario can be made realistic and can address the shortcomings of the SM: nonzero neutrino masses, dark matter (DM), baryon asymmetry of the Universe (BAU), inflation as well as an explication for the smallness of the QCD $\theta$-term.

Here, we show that this can be achieved by simply including three right-handed neutrinos with Majorana masses $M$ below the electroweak (EW) scale. Right-handed neutrinos can account for the observed neutrino oscillations, DM and BAU. We consider a concrete implementation of the softened gravity idea where the Einstein-Hilbert Lagrangian is extended to include all terms quadratic in the curvature as well as a nonminimal coupling $\xi$ between the Higgs and gravity. We address the question of whether this theory might be a candidate UV completion of Einstein gravity.

The same radiatively stable values of the parameters that lead to a natural Higgs mass (found in Ref. [2]) also preserve the smallness of $M$ and $\theta$. The concept of naturalness used here is the one based on finite quantities

Published by the American Physical Society under the terms of the Creative Commons Attribution 4.0 International license. Further distribution of this work must maintain attribution to the author(s) and the published article's title, journal citation, and DOI. (after renormalization), where unphysical power-law divergences with respect to the momentum cutoff are disregarded [3-6].

We also show that a possible gravitational breaking of $C P$ (which could be due to $\theta$ ) produces no visible effects in the observable quantities, thanks to the presence of the right-handed neutrinos. Inflation is mainly due to the effective Starobinsky scalar $z$ [7] (which automatically emerges from the terms quadratic in the curvature), and the Higgs gives very small contributions even in the natural parameter space.

We find it remarkable that all the above-mentioned problems can be solved in such a simple extension of the SM.

This paper is organized as follows. In the next section, we describe the theory of softened gravity that will be considered in detail in this paper. In Sec. III, we discuss its spectrum. The following Sec. IV is dedicated to the study of some quantum aspects. Section V shows the naturalness of the Higgs mass, the QCD $\theta$ angle and right-handed neutrino masses and Yukawa couplings in this context. In the same section, we also discuss the connection between the possible gravitational violation of $C P$ and the neutrino sector. Section VI presents a detailed analysis of inflation, and finally we provide our conclusions and outlook in Sec. VII. We provide technical material in two Appendixes.

\section{THE THEORY}

The full Lagrangian (density) is given by

$$
\mathcal{L}=\sqrt{-g}\left(\mathcal{L}_{\text {gravity }}+\mathcal{L}_{\mathrm{SM}}+\mathcal{L}_{N}\right) .
$$

Here, $\mathcal{L}_{\text {gravity }}$ represents the pure gravitational Lagrangian plus the possible nonminimal coupling between the Higgs and gravity, which, modulo total derivatives [8], is 
$\mathcal{L}_{\text {gravity }}=\frac{R^{2}}{6 f_{0}^{2}}+\frac{\frac{1}{3} R^{2}-R_{\mu \nu}^{2}}{f_{2}^{2}}-\left(\frac{\bar{M}_{\mathrm{Pl}}^{2}}{2}+\xi|H|^{2}\right) R-\Lambda$,

where $f_{2}$ and $f_{0}$ play the role of gravitational couplings, $\bar{M}_{\mathrm{Pl}}$ is the reduced Planck mass and $\Lambda$ is the cosmological constant. $\mathcal{L}_{\mathrm{SM}}$ represents the usual SM Lagrangian, minimally coupled to gravity. $\mathcal{L}_{N}$ is the term that depends on the right-handed neutrinos $N_{i}(i=1,2,3)$,

$\mathcal{L}_{N}=i \bar{N}_{i} \not \partial N_{i}+\left(\frac{1}{2} N_{i} M_{i j} N_{j}+Y_{i j} L_{i} H N_{j}+\right.$ H.c. $)$,

where $M_{i j}$ and $Y_{i j}$ are the elements of the Majorana mass matrix $M$ and the neutrino Yukawa coupling matrix $Y$, respectively.

Note that the new gravitational terms associated with the couplings $f_{0}, f_{2}$ and $\xi$ are necessary for the renormalization of the theory; even if we do not introduce them in the classical theory, radiative corrections generate them.

\section{SPECTRUM}

We now turn to the spectrum. As usual, there is a massless spin-2 graviton. Also, the term $\left(\frac{1}{3} R^{2}-R_{\mu \nu}^{2}\right) / f_{2}^{2}$ in (2.2) corresponds to a ghost: a field with an unusual minus sign in front of its kinetic term. In the present case, this field has spin-2 and mass $M_{2} \equiv f_{2} \bar{M}_{\mathrm{Pl}} / \sqrt{2}$ [9]. In the next section, we will discuss a possible sensible way of interpreting such a field.

The term $R^{2} /\left(6 f_{0}^{2}\right)$ leads instead to the scalar $z$. To see this, one can write the scalar-tensor part of the Lagrangian, $\mathcal{L}_{\text {st }}$, in the Einstein frame [2,10,11],

$\mathcal{L}_{\mathrm{st}}=\sqrt{-g_{E}}\left[\frac{\frac{1}{3} R_{E}^{2}-R_{E \mu \nu}^{2}}{f_{2}^{2}}-\frac{\bar{M}_{\mathrm{Pl}}^{\prime 2}}{2} R_{E}+\mathcal{L}_{\phi}-V_{E}\right]$,

where everything is computed with the new metric

$$
g_{\mu \nu}^{E} \equiv g_{\mu \nu} \times \frac{z^{2}}{6 \bar{M}_{\mathrm{Pl}}^{\prime 2}},
$$

$\bar{M}_{\mathrm{Pl}}^{\prime 2} \equiv \bar{M}_{\mathrm{Pl}}^{2}+2 \xi v^{2}$ (note that $v \simeq 174 \mathrm{GeV}$, and in practice one can take $\left.\bar{M}_{\mathrm{Pl}}^{\prime 2}=\bar{M}_{\mathrm{Pl}}^{2}\right)$ and

$\mathcal{L}_{\phi} \equiv \frac{6 \bar{M}_{\mathrm{Pl}}^{\prime 2}}{z^{2}}\left(\left|D_{\mu} H\right|^{2}+\frac{\left(\partial_{\mu} z\right)^{2}}{2}\right)$,

$V_{E} \equiv \frac{36 \bar{M}_{\mathrm{Pl}}^{\prime 4}}{z^{4}}\left[V(H)+\frac{3 f_{0}^{2}}{8}\left(\frac{z^{2}}{6}-2 \xi|H|^{2}-\bar{M}_{\mathrm{Pl}}^{2}\right)^{2}\right]$.

The potential $V$ is the SM one: $V(H)=\lambda\left(|H|^{2}-v^{2}\right)^{2}+\Lambda$.
The minimum of $V_{E}$ occurs when the Higgs is at the EW scale, $v$, and $z \approx\langle z\rangle \equiv \sqrt{6 \bar{M}_{\mathrm{Pl}}^{2}}$ (here, we neglect tiny corrections due to $\Lambda / \bar{M}_{\mathrm{Pl}} \neq 0$ ). Notice that at $z=\langle z\rangle$ the kinetic terms of the scalars are canonically normalized, and therefore the squared mass matrix for scalars, $\mathcal{M}_{0}^{2}$, is the Hessian matrix of $V_{E}$ computed at this point of minimum. This procedure leads to

$$
\mathcal{M}_{0}^{2} \approx\left(\begin{array}{cc}
M_{0}^{\prime 2} & -\epsilon M_{0}^{\prime} m \\
-\epsilon M_{0}^{\prime} m & \left(1+\epsilon^{2}\right) m^{2}
\end{array}\right),
$$

where $M_{0}^{\prime 2} \equiv f_{0}^{2} \bar{M}_{\mathrm{Pl}}^{\prime 2} / 2, m \equiv 2 \sqrt{\lambda} v$ and $\epsilon \equiv \sqrt{\frac{6}{4 \lambda}} f_{0} \xi$ (note that $\lambda$ is required to be positive by the stability of the potential $V$ ). As usual, when $\lambda<0$ we have a tachyonic instability. For $M_{0}^{\prime} \gg m$, which we expect because $M_{0}^{\prime} \propto \bar{M}_{\mathrm{Pl}}$, the mixing becomes small, and the scalar masses are approximately $M_{0}^{\prime}$ and $m$. We will see in Sec. VI B that this approximation is very accurate.

To analyze the neutrino sector, we take (thanks to the complex Autonne-Takagi factorization) $M$ real and diagonal without loss of generality, $M=\operatorname{diag}\left(M_{1}, M_{2}, M_{3}\right)$, where the $M_{i}$ are real mass parameters. The neutrinos acquire a Dirac mass matrix $m_{D}=v Y$, which can be parametrized as $m_{D}=\left(m_{D 1}, m_{D 2}, m_{D 3}\right)$, where $m_{D i}$ are column vectors. Integrating out the heavy neutrinos $N_{i}$, one then obtains the usual seesaw formula for the light neutrino Majorana mass matrix

$$
m_{\nu}=\frac{m_{D 1} m_{D 1}^{T}}{M_{1}}+\frac{m_{D 2} m_{D 2}^{T}}{M_{2}}+\frac{m_{D 3} m_{D 3}^{T}}{M_{3}} .
$$

By means of a unitary (Autonne-Takagi) redefinition of the left-handed SM neutrinos, we can diagonalize $m_{\nu}$ to obtain the mass eigenvalues $m_{1}, m_{2}$ and $m_{3}$ (the left-handed neutrino Majorana masses). The experimental constraints on neutrino masses and oscillations (see Ref. [12] for recent determinations) can be satisfied by choosing appropriately the unitary matrix $U_{\nu}$ that implements such transformation (the Pontecorvo-Maki-Nakagawa-Sakata matrix) and the $m_{i}$.

Here, the $N_{i}$ are also responsible for DM [13-15] and BAU [14-16]. For example, we find that all bounds to account for neutrino masses and oscillations (within $1 \sigma$ ) [12], for DM and BAU [15], can be satisfied for

$$
M_{1} \sim \mathrm{keV}, \quad M_{2,3} \sim \mathrm{GeV}, \quad\left|Y_{i j}\right|<10^{-7} .
$$

These low-scale right-handed neutrinos can be searched for the laboratory or through astrophysical observations (see for example Refs. $[15,17]$ and references therein). This proposal can therefore be tested.

\section{QUANTUM ASPECTS}

The theory with Lagrangian (2.1) by itself does not eliminate the Landau poles of the SM, which, however, 
occur many orders of magnitude above the Planck scale where no experiments or observations can be made. We will therefore avoid this problem by assuming that there is a minimal length much larger than the Landau pole scales but still much shorter than the Planck length.

The Lagrangian in (2.1) defines a renormalizable theory of gravity [9] (actually, of all interactions). The price to pay is the presence of a ghost (which we have seen in the previous section). Such a field emerges because of the presence of four time derivatives in the Lagrangian [18]. The spectrum of such a theory, however, becomes bounded from below at the quantum level if negative norm states are introduced (see e.g. Ref. [19] and references therein). In particular, the quanta of the ghost field have to have negative norm, while all remaining quanta have positive norm. Recently, Ref. [19] showed (assuming a single fourderivative degree of freedom) that such a quantization can be obtained with normalizable wave functions and a welldefined Euclidean path integral.

The remaining problem of having a sensible probabilistic interpretation of negative-norm states could be bypassed by the Lee-Wick idea [20], which assumes all stable states in the theory to have positive norm. Indeed, theories of this sort are sensible as long as we only look at the energy spectrum and transition probabilities between asymptotic (stable) states ( $S$-matrix elements). Reference [21] argued that the assumption of Lee and Wick is satisfied in the theory of gravity above. One of the purposes of this section is to explicitly prove that the ghost is unstable in this theory.

\section{A. Ghost decay}

Given that the ghost at hand has spin 2, a direct calculation of its decay involves the complications of the corresponding Lorentz indices. For this reason, one would like to use the optical theorem and compute equivalently the imaginary part of the ghost propagator. However, the optical theorem is derived in theories with positive norms only and requires a generalization here, given that the ghost state has negative norm.

To obtain such a generalization consider the time evolution operator $U$, which is defined as usual as the linear operator that transforms the state at the initial time into the state at the generic time. The usual procedure is to define the operator $T$ by

$$
U \equiv 1+i T
$$

By using the unitarity condition (which is fulfilled even in the presence of negative norms [19]), we obtain

$$
i\left(T^{\dagger}-T\right)=T^{\dagger} T .
$$

If we now take the matrix element between an initial state $|i\rangle$ and a final state $|f\rangle$, we find

$$
i\left(T_{f i}^{\dagger}-T_{f i}\right)=\left(T^{\dagger} T\right)_{f i},
$$

where $\quad T_{f i} \equiv\langle f|T| i\rangle, \quad T_{f i}^{\dagger} \equiv\left\langle f\left|T^{\dagger}\right| i\right\rangle \quad$ and $\quad\left(T^{\dagger} T\right)_{f i} \equiv$ $\left\langle f\left|T^{\dagger} T\right| i\right\rangle$. In theories with positive norms only, the completeness relation is $1=\sum_{n}|n\rangle\langle n|$, where $\{|n\rangle\}$ is an orthonormal basis, $\left\langle n^{\prime} \mid n\right\rangle=\delta_{n^{\prime} n}$. In the presence of both negative and positive norms, however, the scalar product between two generic states $|\alpha\rangle$ and $|\beta\rangle$ can be written as

$$
\langle\beta \mid \alpha\rangle=(\beta, \eta \alpha),
$$

where (...) is a positively defined scalar product and $\eta$ is assumed to be a diagonalizable operator with eigenvalues +1 and -1 [22], so $\eta^{2}=1$. The completeness relation now reads $\eta=\sum_{n}|n\rangle\langle n|$, so

$$
1=\eta^{2}=\sum_{n}|n\rangle\left\langle n\left|\eta=\sum_{n}\right| n\right\rangle\langle n| \eta_{n},
$$

where we have constructed the basis $\{|n\rangle\}$ with the eigenvectors of $\eta$. By inserting this into Eq. (4.3), we get

$$
i\left(T_{f i}^{\dagger}-T_{f i}\right)=\sum_{n} \eta_{n} T_{n f}^{*} T_{n i},
$$

which is the generalization of the optical theorem we were looking for.

To apply this formula to compute a decay, we set the initial and final states equal to each other $(|i\rangle=|f\rangle=|\alpha\rangle)$, and so

$$
2 \operatorname{Im}\left(T_{\alpha \alpha}\right)=\sum_{n} \eta_{n}\left|T_{n \alpha}\right|^{2} .
$$

Notice that $\left|T_{n \alpha}\right|^{2}=\left|U_{n \alpha}-\langle n \mid \alpha\rangle\right|^{2}$, where $U_{n \alpha} \equiv\langle n|U| \alpha\rangle$. We are interested here in the decay of a negative norm particle; we therefore focus on states $|\alpha\rangle$ that are normalizable and set $\langle\alpha \mid \alpha\rangle= \pm 1$. We would like to apply Eq. (4.7) within perturbation theory at first order, so we can take $T_{\alpha \alpha}=0$ and $\eta_{n}=1$ in the right-hand side; the former equality holds because at zero order $T$ does not transform the ghost into itself or into itself plus additional states (for kinematical reasons), and the latter holds because the ghost is the only state with negative norm. Then, $\left|T_{n \alpha}\right|^{2}$ is the transition probability ${ }^{1}$; for the process $\alpha \rightarrow n$, and the righthand side of Eq. (4.7) is the total probability that the state $|\alpha\rangle$ decays.

The next step to compute the ghost decay is to rewrite the four-derivative terms as two-derivatives where the ghost field is explicit in the Lagrangian. This is the analogue of what we have done in Sec. III to have the field of the scalar $z$ explicit and has been done in Ref. [23]. In this case, the

\footnotetext{
${ }^{1}$ In the present work, we define the probability as the absolute value squared of the amplitude. One should keep in mind, however, that other definitions are possible [19].
} 
trick is to introduce a rank-2 Lagrange multiplier $\pi_{\mu \nu}$, which is explicitly defined in Ref. [23]. We eliminate the linear mixing between $\pi_{\mu \nu}$ and the fluctuation of the metric $h_{\mu \nu}$ around the flat space (as well as with all the other fields) by introducing

$$
\bar{h}_{\mu \nu} \equiv h_{\mu \nu}+\pi_{\mu \nu}+\sqrt{\frac{2}{3}} \frac{\delta z}{\bar{M}_{\mathrm{Pl}}} \eta_{\mu \nu},
$$

where $\delta z \equiv z-\langle z\rangle$. The field $\bar{h}_{\mu \nu}$ corresponds to the usual massless graviton, $\pi_{\mu \nu}$ represents the ghost, and $\delta z$ represents the quantum scalar field associated with the fluctuations of $z$ around its vacuum expectation value.

We also find

$$
\begin{aligned}
\left(\square-M_{2}^{2}\right) \pi_{\mu \nu} & =0, \quad \partial^{\mu} \pi_{\mu \nu}=0, \\
\pi_{\mu}{ }^{\mu} & =0 \quad \text { (at the linearized level), }
\end{aligned}
$$

where the indices are raised and lowered here with $\eta_{\mu \nu}$. This confirms that $\pi_{\mu \nu}$ is a massive spin-2 field and can be expanded as follows,

$$
\begin{aligned}
\pi_{\mu \nu}(x)= & \frac{2}{\bar{M}_{\mathrm{Pl}}} \sum_{i, \vec{k}} \frac{1}{\sqrt{2 V \omega_{\pi}(\vec{k})}}\left(e_{\mu \nu}^{i}(\vec{k}) a_{\pi i}(\vec{k}) e^{-i k x}\right. \\
& \left.+\bar{e}_{\mu \nu}^{i}(\vec{k}) a_{\pi i}^{\dagger}(\vec{k}) e^{i k x}\right),
\end{aligned}
$$

where $V$ is the space volume (that should be taken to $\infty$ at the end) and $\omega_{\pi}(\vec{k})=\sqrt{M_{2}^{2}+\vec{k}^{2}}$ and $e_{\mu \nu}^{i}(\vec{k})$ are $^{2}$ the polarization tensors corresponding to the wave number $\vec{k}$; the index $i$ labels the helicity state. The operators $a_{\pi i}(\vec{k})$ and $a_{\pi i}^{\dagger}(\vec{k})$ are annihilation and creation operators, respectively, and fulfill the commutation relations

$$
\left[a_{\pi i}(\vec{k}), a_{\pi j}\left(\vec{k}^{\prime}\right)\right]=0, \quad\left[a_{\pi i}(\vec{k}), a_{\pi j}\left(\vec{k}^{\prime}\right)^{\dagger}\right]=-\delta_{i j} \delta_{\vec{k} \vec{k}^{\prime}} .
$$

Note the minus sign on the right-hand side, which has been introduced because we are dealing with ghosts (see Ref. [19]).

The $e_{\mu \nu}^{i}(\vec{k})$ transform covariantly as a rank-2 tensor. Notice that the second equation in (4.9) gives

$$
p^{\mu} e_{\mu \nu}^{i}(\vec{p})=0 .
$$

In the massive spin-2 case, we are considering there are five helicity states (so $i=1,2,3,4,5$ ). In this case, one can easily obtain the $e_{\mu \nu}^{i}(\vec{p})$ in the rest frame, $p_{\mu}=\left(M_{2}, 0,0,0\right)$, and then use general Lorentz boosts

\footnotetext{
${ }^{2} \bar{e}_{\pi \mu \nu}^{i}(\vec{k})$ represents the corresponding complex conjugate object.
}

to obtain the polarization tensors in an arbitrary frame. In the rest frame, Eq. (4.12) becomes $e_{0 \mu}^{i}=0$, which, together with the traceless condition $e_{\mu}^{i \mu}=0$ [the third equation in (4.9)], can be fulfilled by the basis given in Appendix A. One can directly check that

$$
e_{\mu \nu}^{i}(\vec{p}) e^{j \mu \nu}(\vec{p})=\delta^{i j}
$$

and

$$
\sum_{i=1}^{5} e_{\mu \nu}^{i}(\vec{p}) \bar{e}_{\rho \sigma}^{i}(\vec{p})=P_{\mu \nu \rho \sigma}^{(2)}(p),
$$

where

$$
P_{\mu \nu \rho \sigma}^{(2)}=\frac{1}{2} T_{\mu \rho} T_{\nu \sigma}+\frac{1}{2} T_{\mu \sigma} T_{\nu \rho}-\frac{1}{3} T_{\mu \nu} T_{\rho \sigma},
$$

and $T_{\mu \nu}(p)=\eta_{\mu \nu}-p_{\mu} p_{\nu} / p^{2}$. The equality in (4.14) can be shown by considering first the rest frame, where the $e_{\mu \nu}^{i}$ are simple and explicitly given above, and then noticing that both $\sum_{i=1}^{5} e_{\mu \nu}^{i}(\vec{p}) \bar{e}_{\rho \sigma}^{i}(\vec{p})$ and $P_{\mu \nu \rho \sigma}^{(2)}(p)$ are rank-4 tensors and so they coincide if they are equal in a given frame.

Finally, by using the generalized optical theorem in Eq. (4.7), we obtain that the decay rate $\Gamma$ of the gravitational ghost state with momentum $p_{\mu}$ is

$$
\Gamma=\left(\frac{2}{\bar{M}_{\mathrm{Pl}}}\right)^{2} \frac{1}{p_{0}} \frac{1}{5} P_{\mu \nu \rho \sigma}^{(2)}(p) \operatorname{Im} \Pi^{\mu \nu \rho \sigma}(p),
$$

where $\Pi^{\mu \nu \rho \sigma}$ is the amputated loop Feynman amplitude (multiplied by $-i$ ). The factor $1 / 5$ appears because we have averaged with respect to the initial polarization.

That this decay rate is not zero can be explicitly checked by considering the decay into two real scalars with mass $m$, for instance two Higgs in the final state. In this case, we find

$$
\begin{aligned}
& \Gamma(\text { ghost } \rightarrow \text { scalar scalar }) \\
& \quad=\frac{\left(M_{2}^{2}-4 m^{2}\right)^{2} \operatorname{Im} B_{0}\left(M_{2}^{2}, m^{2}, m^{2}\right)}{60(4 \pi)^{2} p_{0} \bar{M}_{\mathrm{Pl}}^{2}},
\end{aligned}
$$

where the function $B_{0}$ is defined in Appendix B, Eq. (B2). The same Appendix also shows

$$
\operatorname{Im} B_{0}\left(p^{2}, m^{2}, m^{2}\right)=\pi \sqrt{1-\frac{4 m^{2}}{p^{2}}} \theta\left(p^{2}-4 m^{2}\right) .
$$

By using this result, we find

$$
\begin{aligned}
& \Gamma(\text { ghost } \rightarrow \text { scalar scalar }) \\
& \quad=\frac{\left(M_{2}^{2}-4 m^{2}\right)^{2} \sqrt{1-\frac{4 m^{2}}{p^{2}}} \theta\left(M_{2}^{2}-4 m^{2}\right)}{960 \pi p_{0} \bar{M}_{\mathrm{Pl}}^{2}} .
\end{aligned}
$$


This expression can be simplified by going to the ghost rest frame $p=\left(M_{2}, 0,0,0\right)$ and assuming $M_{2} \gg 2 m$,

$\Gamma($ ghost $\rightarrow$ scalar scalar $)$

$=\frac{M_{2}^{3}}{960 \pi \bar{M}_{\mathrm{Pl}}^{2}}\left(\right.$ in the ghost rest frame and for $\left.M_{2} \gg 2 m\right)$.

Of course, the interactions of $\pi_{\mu \nu}$ with the other fields of the theory under study cannot slow the decay, but they make it faster. This definitely shows that the ghost is unstable and the Lee-Wick idea may be implemented. Other challenges that have to be faced before considering this theory a completely satisfactory UV completion of Einstein's gravity will be mentioned in Sec. VII.

\section{B. RGEs and threshold effects}

In order to address naturalness issues in this model (see Sec. V), we need the renormalization group equations (RGEs); they encode the leading quantum corrections. The one-loop RGEs for the dimensionless couplings for energies above all mass thresholds are (see e.g. Refs. [2,24-26] and references therein)

$$
\begin{gathered}
\frac{d f_{2}^{2}}{d t}=-\frac{109}{6} f_{2}^{4}, \\
\frac{d f_{0}^{2}}{d t}=\frac{5}{3} f_{2}^{4}+5 f_{2}^{2} f_{0}^{2}+\frac{5}{6} f_{0}^{4}+f_{0}^{4} \frac{(1+6 \xi)^{2}}{3}, \\
\frac{d \xi}{d t}=(1+6 \xi)\left(y_{t}^{2}-\frac{3}{4} g_{2}^{2}-\frac{3}{20} g_{1}^{2}+2 \lambda+\frac{1}{3} \operatorname{Tr}\left(Y^{\dagger} Y\right)\right) \\
+\frac{f_{0}^{2}}{3} \xi(1+6 \xi)(2+3 \xi)-\frac{5}{3} \frac{f_{2}^{4}}{f_{0}^{2}} \xi, \\
\frac{d g_{1}^{2}}{d t}=\frac{41 g_{1}^{4}}{5}, \quad \frac{d g_{2}^{2}}{d t}=-\frac{19 g_{2}^{4}}{3}, \quad \frac{d g_{3}^{2}}{d t}=-14 g_{3}^{4}, \\
\frac{d y_{t}^{2}}{d t}=y_{t}^{2}\left(9 y_{t}^{2}-16 g_{3}^{2}-\frac{9 g_{2}^{2}}{2}-\frac{17 g_{1}^{2}}{10}+2 \operatorname{Tr}\left(Y^{\dagger} Y\right)+\frac{15}{4} f_{2}^{2}\right), \\
\frac{d Y}{d t}=Y\left[3 y_{t}^{2}-\frac{9}{20} g_{1}^{2}-\frac{9}{4} g_{2}^{2}+\frac{3}{2} Y^{\dagger} Y+\operatorname{Tr}\left(Y^{\dagger} Y\right)+\frac{15}{8} f_{2}^{2}\right],
\end{gathered}
$$

$$
\begin{aligned}
\frac{d \lambda}{d t}= & \left(24 \lambda+12 y_{t}^{2}-\frac{9 g_{1}^{2}}{5}-9 g_{2}^{2}+4 \operatorname{Tr}\left(Y^{\dagger} Y\right)\right. \\
& \left.+5 f_{2}^{2}+f_{0}^{2}(1+6 \xi)^{2}\right) \lambda \\
& -6 y_{t}^{4}+\frac{9 g_{2}^{4}}{8}+\frac{27 g_{1}^{4}}{200}+\frac{9 g_{2}^{2} g_{1}^{2}}{20}-2 \operatorname{Tr}\left(\left(Y^{\dagger} Y\right)^{2}\right) \\
& +\frac{\xi^{2}}{2}\left(5 f_{2}^{4}+f_{0}^{4}(1+6 \xi)^{2}\right),
\end{aligned}
$$

where $t=\ln \left(\bar{\mu} / \overline{\mu_{0}}\right) /(4 \pi)^{2}, \bar{\mu}$ is the $\overline{\mathrm{MS}}$ renormalization scale $^{3}$ and $\bar{\mu}_{0}$ is a reference energy. Here, we have ignored the Yukawa couplings of the SM that are smaller than the top Yukawa, $y_{t}$, and the $g_{i}$ are the gauge couplings.

Going below the mass thresholds $M_{2}$ and $M_{0}^{\prime}$, one can neglect the contributions due to $f_{2}$ and $f_{0}$, respectively. One can wonder whether the scalar threshold due to $z$ induces a tree-level shift of the quartic Higgs coupling, along the lines of Ref. [27]. We now show that such a shift is negligible. This effect can emerge because setting the heavy scalar (in this case, $\delta z \equiv z-\langle z\rangle$ ) equal to zero is not compatible with the equations of motion. This occurs if there are scalar couplings of the schematic form (heavy) $\mathrm{x}$ (light) x (light) in the Lagrangian (in this case, $\delta z \delta h^{2}$, where $\delta h \equiv h-\sqrt{2} v$ ). Using Eq. (3.3) leads to such a coupling, $\sim f_{0}^{2} \xi \bar{M}_{\mathrm{Pl}} \delta z \delta h^{2}$ (modulo order 1 factors and neglecting contributions suppressed by $v^{2} / \bar{M}_{\mathrm{Pl}}^{2}$ and the tiny value of $\Lambda$ ). The shift $\delta \lambda$ in the quartic coupling is given by the square of the coefficient of the (heavy) $x$ (light) $x$ (light) term, in this case, $\sim f_{0}^{2} \xi \bar{M}_{\mathrm{PI}}$ times the propagator of $z$ at zero external momentum [27]: $\delta \lambda \sim f_{0}^{2} \xi^{2}$. In Secs. V and VI, we will see that the requirement of successful inflation and Higgs mass naturalness implies $f_{0} \sim 10^{-5}$ and $\xi \approx-1 / 6$, so that $f_{0}^{2} \xi^{2} \sim 10^{-11}$ and this effect is negligibly small.

As far as the RGEs of the mass parameters are concerned, we find that $m^{2}, M$ and $\bar{M}_{\mathrm{Pl}}^{2}$ obey

$$
\begin{aligned}
\frac{d m^{2}}{d t}= & m^{2}\left(12 \lambda+6 y_{t}^{2}-\frac{9}{2} g_{2}^{2}-\frac{9}{10} g_{1}^{2}+2 \operatorname{Tr}\left(Y^{\dagger} Y\right)\right. \\
& \left.+5 f_{2}^{2}+f_{0}^{2}(1+6 \xi)+6 \xi^{2}+2 G\right) \\
& +8 \operatorname{Tr}\left(Y^{\dagger} Y M^{\dagger} M\right)-5 f_{2}^{4} \xi \bar{M}_{\mathrm{Pl}}^{2}-f_{0}^{4} \xi(1+6 \xi) \bar{M}_{\mathrm{Pl}}^{2} \\
\frac{d M}{d t}= & M Y^{\dagger} Y+\left(Y^{\dagger} Y\right)^{T} M+\frac{15}{8} f_{2}^{2} M+M G \\
\frac{d \bar{M}_{\mathrm{Pl}}^{2}}{d t}= & -\frac{2}{3} m^{2}+\frac{1}{24} \operatorname{Tr}\left(M^{\dagger} M\right)-4 \xi m^{2} \\
& +\left(\frac{2 f_{0}^{2}}{3}-\frac{5 f_{2}^{4}}{3 f_{0}^{4}}+2 G\right) \bar{M}_{\mathrm{Pl}}^{2}
\end{aligned}
$$

Here, $G$ is a gauge-dependent quantity; for example, using the same gauge fixing action as in Ref. [2],

$$
\begin{aligned}
S_{\mathrm{gf}} & =-\frac{1}{2 \xi_{g}} \int d^{4} x f_{\mu} \partial^{2} f_{\mu}, \\
f_{\mu} & =\partial_{\nu}\left(h_{\mu \nu}-c_{g} \frac{1}{2} \eta_{\mu \nu} h_{\alpha \alpha}\right),
\end{aligned}
$$

\footnotetext{
${ }^{3}$ All renormalized couplings in this work are defined in the $\overline{\mathrm{MS}}$ scheme.
} 
where $h_{\mu \nu}=g_{\mu \nu}-\eta_{\mu \nu}$, leads to

$$
G=\frac{\left(3 c_{g}^{2}-12 c_{g}+13\right) \xi_{g}}{4\left(c_{g}-2\right)}+\frac{3\left(c_{g}-1\right)^{2} f_{0}^{2}}{4\left(c_{g}-2\right)^{2}} .
$$

The gauge dependence cancels as it should in the RGEs of $M / \bar{M}_{\mathrm{Pl}}$ and $m^{2} / \bar{M}_{\mathrm{Pl}}^{2}$.

\section{NATURALNESS}

Notice that the $\beta$-function in (4.27) vanishes as $M \rightarrow 0$. Therefore, by starting from small values [such as those in (3.5)] at low energy, one does not end up with a much larger $M$. This occurs because $M$ breaks lepton symmetry, while all other fields (gravity included) preserve it. Such small values of $M$ are therefore natural even taking into account gravity. This is because our softened gravity preserves global lepton symmetry.

The same is true for $Y$, given the structure of the $\beta$ function in (4.24). As a result, the naturalness of the EW scale $m$ leads to the same conditions obtained in Ref. [2] [as it can be seen from Eq. (4.26)]: the order of magnitude of $f_{2}^{4}$ and $f_{0}^{4}(1+6 \xi)$ should not exceed $M_{h}^{2} / \bar{M}_{\mathrm{Pl}}^{2}$. This condition is preserved by the renormalization group $(\mathrm{RG})$ running [see Eqs. (4.21), (4.22) and (4.23)]. The smallness of these couplings corresponds to the softening of gravity. Notice that one important ingredient to ensure this result is the fact that the small values of $M$ and $Y$ [see for example (3.5)] ensures that neutrinos do not give unnaturally large corrections to the Higgs mass. This is opposed to the standard leptogenesis scenario [28], which occurs through the decay of very heavy [29] right-handed neutrinos and can introduce a fine-tuning in the Higgs mass [4].

In a similar way, we also show now that the smallness of the $\theta$-term is natural in this context. In the SM, the $\beta$ function of $\theta$ starts at least at seven loops and is at most of order $10^{-15}$ [30]. This is because one needs to construct a flavor invariant $C P$-breaking term out of the quark Yukawa couplings. Therefore, in the SM, the running of $\theta$ is negligibly small. The right-handed neutrino sector contains other sources of $C P$ breaking and can potentially introduce a larger running. However, in order to connect the $\theta$-vertex with a right-handed neutrino, you need three loops (you should insert a quark, a Higgs and a right-handed neutrino). This leads to a $1 /(4 \pi)^{6}$ suppression. Moreover, you have at least an extra factor that is quadratic in the $Y_{i j}$, which has to be very small given that all right-handed neutrinos are below the EW scale [see e.g. Eq. (3.5)]. Therefore, also the right-handed neutrino sector preserves the smallness of the $\theta$-running. Finally, notice that gravity, given that it is softened and $C P$-preserving in our context, does not reintroduce a sizable running.

It is now a good point to comment on the possible $C P$ breaking extension of the softened gravity theory at hand. Given that we limit to terms in the Lagrangian which are at most quadratic in the curvature, we could add a "gravitational $\theta$-term,"

$$
\epsilon^{\mu \nu \rho \sigma} R_{\mu \nu}^{\alpha \beta} R_{\rho \sigma \alpha \beta}
$$

which may potentially affect the observable predictions of the theory. This term (as well as some phases in the quark mass matrix) could be (partially) due to removing the QCD $\theta$-term via a chiral transformation of the quarks [31]. Since it can be rotated away with an anomalous chiral transformation, its coefficient in the Lagrangian cannot be much larger than $2 \pi$ times $1 /(4 \pi)^{2}$. For such a small value, the gravitational $\theta$-term could only affect very energetic phenomena, such as inflation. Moreover, the presence of right-handed neutrinos helps; one can perform a $\mathrm{U}(1)$ transformation of $N_{i}$, that is $N_{j} \rightarrow \exp (i \beta) N_{j}$, where $\beta$ is a real number, which removes completely such a gravitational term and, as side effect, only rescales $M_{j}$ and $Y_{i j}$ in the following way: $M_{j} \rightarrow \exp (2 i \beta) M_{j}, Y_{i j} \rightarrow$ $\exp (i \beta) Y_{i j}$. This rescaling produces no effect in neutrino observables, which can therefore be compatible with data. This is because the neutrino mass matrix in (3.4) is invariant under such a transformation.

\section{INFLATION}

Let us finally turn to inflation. In a similar context, Refs. [10,11,32] showed that inflation is mainly triggered by the Starobinsky effective scalar $z$, rather than the Higgs. We show that the same happens here even in the natural parameter space.

\section{A. Multifield inflation formalism}

Although the theory studied here has only two scalar fields (in the unitary gauge), it is convenient to start from a formalism suitable for a general number of scalars (as the equations will be shorter). This formalism has been studied in the past [33], but we will obtain weaker slow-roll conditions; therefore, we present the explicit derivation here. We also take advantage to elucidate some points in multifield inflation.

We rewrite the scalar-tensor Lagrangian in (3.1) as

$$
\begin{aligned}
\mathcal{L}_{\text {st }}= & \sqrt{-g_{E}}\left[\frac{\frac{1}{3} R_{E}^{2}-R_{E \mu \nu}^{2}}{f_{2}^{2}}-\frac{\bar{M}_{\mathrm{Pl}}^{\prime 2}}{2} R_{E}\right. \\
& \left.+\frac{K_{i j}(\phi)}{2} \partial_{\mu} \phi^{i} \partial^{\mu} \phi^{j}-U(\phi)\right],
\end{aligned}
$$

where the field metric $K_{i j}$ and the potential $U$ are generic functions of the scalar fields $\phi^{i}$. In our case, we are interested in the case $\phi^{i}=\{z, h\}$, where $h$ is the physical Higgs field, but we keep the formalism general as explained above. 
Next, we consider the Friedmann-Robertson-Walker (FRW) metric.

$$
\begin{aligned}
d s_{E}^{2} & \equiv g_{\mu \nu}^{E} d x^{\mu} d x^{\nu} \\
& =d t^{2}-a(t)^{2}\left[\frac{d r^{2}}{1-k r^{2}}+r^{2}\left(d \Theta^{2}+\sin ^{2} \Theta d \phi^{2}\right)\right] .
\end{aligned}
$$

We neglect from now on the curvature contribution $k$ as during inflation the energy density is dominated by the scalar fields. Then, the Einstein equations (EEs) and the scalar equations imply the following equations for $a(t)$ and the spatially homogeneous fields $\phi^{i}(t)$,

$$
\begin{gathered}
H^{2}=\frac{K_{i j} \dot{\phi}^{i} \dot{\phi}^{j} / 2+U}{3 \bar{M}_{\mathrm{Pl}}^{\prime 2}}, \quad(t t \text {-component of EEs }) \\
2 a(t) \ddot{a}(t)+\dot{a}(t)^{2}=\frac{a^{2}\left(K_{i j} \dot{\phi}^{i} \dot{\phi}^{j} / 2-U\right)}{\bar{M}_{\mathrm{Pl}}^{\prime 2}\left(k r^{2}-1\right)}, \\
(r r \text {-component of EEs }) \\
2 a(t) \ddot{a}(t)+\dot{a}(t)^{2}=\frac{a^{2}\left(U-K_{i j} \dot{\phi}^{i} \dot{\phi}^{j} / 2\right)}{\bar{M}_{\mathrm{Pl}}^{\prime 2}}, \\
2 a(t) \ddot{a}(t)+\dot{a}(t)^{2}=\frac{(\theta \theta \text {-component of EEs })}{a^{2}\left(U-K_{i j} \dot{\phi}^{i} \dot{\phi}^{j} / 2\right)} \\
\bar{M}_{\mathrm{Pl}}^{\prime 2} \\
(\phi \phi \text {-component of EEs }) \\
\ddot{\phi}^{i}+\gamma_{j k}^{i} \dot{\phi}^{j} \dot{\phi}^{k}+3 H \dot{\phi}^{i}+U^{i}=0, \\
(\text { scalar field equation }),
\end{gathered}
$$

where $H \equiv \dot{a} / a$ and a dot denotes a derivative with respect to $t$. Also, for a generic function $F$ of the scalar fields, we defined $F_{, i} \equiv \partial F / \partial \phi^{i}$, the affine connection $\gamma_{j k}^{i}$ in the scalar field space is

$$
\gamma_{j k}^{i} \equiv \frac{K^{i l}}{2}\left(K_{l j, k}+K_{l k, j}-K_{j k, l}\right),
$$

and $K^{i j}$ denotes the inverse of the field metric (which is used to raise and lower the scalar indices $i, j, k, \ldots$ ); for example $F^{, i} \equiv K^{i j} F_{, j}$. The $r r$-, $\theta \theta$ - and $\phi \phi$-components of the EEs are only one independent equation, and thus the EEs can be simplified to

$$
\begin{gathered}
H^{2}=\frac{K_{i j} \dot{\phi}^{i} \dot{\phi}^{j} / 2+U}{3 \bar{M}_{\mathrm{Pl}}^{2}}, \\
\dot{H}=-\frac{K_{i j} \dot{\phi}^{i} \dot{\phi}^{j}}{2 \bar{M}_{\mathrm{Pl}}^{2}} .
\end{gathered}
$$

Notice that the term suppressed by $f_{2}^{2}$ in the Lagrangian has no effect because it is equal to (modulo total derivatives) the square of the Weyl tensor which vanishes on the FRW metric. We assume that term has no effect on inflation at the quantum level either.

\section{Slow-roll approximation}

We now describe the slow-roll approximation within this formalism. The scalar fields roll slowly down the potential when

$$
\begin{aligned}
K_{i j} \dot{\phi}^{i} \dot{\phi}^{j} & \ll U, \quad\left|\ddot{\phi}^{i}+\gamma_{j k}^{i} \dot{\phi}^{j} \dot{\phi}^{k}\right| \ll 3 H \dot{\phi}^{i}, \\
\left|\ddot{\phi}^{i}+\gamma_{j k}^{i} \dot{\phi}^{j} \dot{\phi}^{k}\right| & \ll U^{i} .
\end{aligned}
$$

Then, from Eqs. (6.3) and (6.7), we obtain

$$
H^{2} \simeq \frac{U}{3 \bar{M}_{\mathrm{Pl}}^{2}}, \quad \dot{\phi}^{i} \simeq-\frac{U^{, i}}{3 H}
$$

(in our notation, $U^{i} \equiv K^{i j} U_{, j}$, where $U_{, i} \equiv \partial U / \partial \phi^{i}$ ). By using (6.12) in the first condition in (6.11), we obtain the first slow-roll condition

$$
\epsilon \equiv \frac{\bar{M}_{\mathrm{Pl}}^{2} U_{, i} U^{, i}}{2 U^{2}} \ll 1
$$

Equations (6.12) tell us

$$
\frac{\dot{H}}{H^{2}} \simeq-\epsilon,
$$

which is guaranteed to be small by (6.13). From (6.12), we find

$$
\begin{aligned}
\frac{\ddot{\phi}^{i}+\gamma_{j k}^{i} \dot{\phi}^{j} \dot{\phi}^{k}}{H \dot{\phi}^{i}} & \simeq-\frac{\dot{H}}{H^{2}}-\frac{\bar{M}_{\mathrm{Pl}}^{2} U_{; j}^{i} U^{, j}}{U U^{, i}} \\
& \simeq-\frac{\bar{M}_{\mathrm{Pl}}^{2} U_{; j}^{i i} U^{, j}}{U U^{, i}}, \quad(i \text { not summed }),
\end{aligned}
$$

where for a generic vector $V^{i}$ on the scalar field space we defined the covariant derivative $V_{; j}^{i} \equiv \partial V^{i} / \partial \phi^{j}+\gamma_{j k}^{i} V^{k}$. Notice that in the formula above the index $i$ is not summed and in the last step we have neglected $\dot{H} / H^{2}$ that we have just proven to be small. Therefore, from (6.11), we obtain the second slow-roll condition

$\left|\frac{\eta_{j}^{i} U^{, j}}{U^{, i}}\right| \ll 1 \quad(i$ not summed $), \quad$ where $\eta_{j}^{i} \equiv \frac{\bar{M}_{\mathrm{Pl}}^{2} U_{; j}^{; i}}{U}$

It is easy to check that $\epsilon$ and $\eta_{j}^{i}$ reduce to the well-known single field slow-roll parameters in the presence of only one 
field. The second slow-roll condition is weaker than the one found in Ref. [33] where it is assumed $\left|\eta_{j}^{i}\right|<1$ and $U^{, j} / U^{, i}$ of order 1.

Combining the two equations in (6.12), we obtain the following dynamical system for $\phi^{i}$,

$$
\dot{\phi}^{i}=-\frac{\bar{M}_{\mathrm{Pl}} U^{, i}(\phi)}{\sqrt{3 U(\phi)}},
$$

which can be solved with a condition at some initial time $t_{0}$, that is $\phi^{i}\left(t_{0}\right)=\phi_{0}^{i}$. Once the functions $\phi^{i}(t)$ are known, we can obtain $H(t)$ from the first equation in (6.12). Let us introduce the number of $e$-folds $N$ by

$$
N\left(\phi_{0}\right) \equiv \int_{t_{e}}^{t_{0}\left(\phi_{0}\right)} d t^{\prime} H\left(t^{\prime}\right)
$$

where $t_{e}$ is the time when inflation ends. Dropping the label on $t_{0}$ and $\phi_{0}$ as they are generic values, we have

$$
N(\phi) \equiv \int_{t_{e}}^{t(\phi)} d t^{\prime} H\left(t^{\prime}\right) .
$$

Notice that we write that $t$ is a function of $\phi$; this is because once the initial position $\phi$ in field space is fixed the time required to go from $\phi$ to the field value when inflation ends is fixed, too, because the dynamical system in (6.17) is of the first order. Note, however, that $H$ also generically depends on $\phi$. Definition (6.19) implies

$$
\frac{d N}{d t}=H
$$

which can be used in (6.12) to obtain a simpler dynamical system for $\phi^{i}$ where the independent variable is $N$ instead of $t$ :

$$
\frac{d \phi^{i}}{d N}=-\frac{\bar{M}_{\mathrm{Pl}}^{2} U^{, i}(\phi)}{U(\phi)} .
$$

\section{Observable predictions}

One can then extract predictions for observable quantities such as the power spectrum $P_{\mathcal{R}}(k)$ of scalar fluctuations, the spectral index $n_{s}$ and the tensor-to-scalar ratio $r$. The measured values at $k=0.002 \mathrm{Mpc}^{-1}$ are $P_{\mathcal{R}}(k)=$ $(2.14 \pm 0.05) \times 10^{-9} \quad[34], n_{s}=0.965 \pm 0.006 \quad[34,35]$ and $r<0.09$ [34]. The power spectrum $P_{\mathcal{R}}(k)$ is (see Eq. (40) of Ref. [36])

$$
P_{\mathcal{R}}(k)=\left(\frac{H}{2 \pi}\right)^{2} N_{, i} N^{, i},
$$

computed at horizon exit $k=a H$. The spectral index $n_{s}$ of scalar perturbations can be computed as

$$
n_{s}=1+\frac{d \ln P_{\mathcal{R}}}{d \ln k} .
$$

By now using $d \ln k=d \ln a H \simeq H d t$, where we noticed that during a nearly exponential expansion $\dot{a} / a \simeq \ddot{a} / \dot{a}$, and Eq. (6.22), we find

$$
n_{s} \simeq 1+2 \frac{\dot{H}}{H^{2}}+2 \frac{N^{, i} \dot{N}_{, i}}{H N_{, j} N^{, j}}
$$

where $N$ is the quantity defined in (6.19). The second term on the right-hand side can be substituted by $-2 \epsilon$ [Eq. (6.14)], while the third one can be computed by using

$$
\begin{aligned}
\dot{N}_{, i}= & \dot{N}_{; i}=\dot{\phi}^{j} N_{; i, j}=\left(\dot{\phi}^{j} N_{, j}\right)_{; i}-\left(\dot{\phi}^{j}\right)_{; i} N_{, j} \\
= & H: i-N^{j}\left(\dot{\phi}_{j}\right)_{; i}, \\
& \left(\dot{\phi}_{j}\right)_{; i} \simeq-\left(\frac{U_{, j}}{3 H}\right)_{; i}=\frac{H_{, i} U_{, j}}{3 H^{2}}-\frac{U_{; j ; i}}{3 H} .
\end{aligned}
$$

This leads to

$$
n_{s}=1-2 \epsilon-\frac{2}{\bar{M}_{\mathrm{Pl}}^{\prime 2} N_{, i} N^{, i}}+\frac{2 \eta_{i j} N^{, i} N^{, j}}{N_{, k} N^{, k}},
$$

where we used (6.12). This formula does not contain a term with the Riemann tensor, unlike Eq. (42) of Ref. [36],

$$
\begin{aligned}
n_{s}= & 1+\frac{2 \dot{H}}{H^{2}}-\frac{2}{\bar{M}_{\mathrm{Pl}}^{\prime 2} N_{, i} N^{, i}}+\frac{2 \eta_{i j} N^{, i} N^{, j}}{N_{, k} N^{, k}} \\
& -\frac{2 \bar{M}_{\mathrm{Pl}}^{\prime 4} R_{i j k l} N^{, i} N^{, l} U^{, j} U^{, k}}{3 N_{, m} N^{, m} U^{2}},
\end{aligned}
$$

because the slow-roll Eqs. (6.12) have been used to evaluate $\left(\dot{\phi}_{j}\right)_{; i}$ in Eq. (6.26).

Also, the tensor-to-scalar ratio can now be easily computed by using a textbook formula for the tensor power spectrum

$$
P_{t}(k)=\frac{2}{\bar{M}_{\mathrm{Pl}}^{2}}\left(\frac{H}{2 \pi}\right)^{2}
$$

to obtain

$$
r \equiv \frac{4 P_{t}(k)}{P_{\mathcal{R}}(k)}=\frac{8}{\bar{M}_{\mathrm{Pl}}^{2} N_{, i} N^{, i}}
$$

\section{B. Higgs-Starobinsky system}

We now apply the formalism of Sec. VI A to the HiggsStarobinsky system defined by (3.1) and (3.3).

A qualitative analysis of the potential $U=V_{E}$ shows that inflation is mainly triggered by $z$ because, even if $\lambda>0$, namely the case in which Higgs inflation [37] is possible [38-40], the fields $\{z, h\}$ are rapidly attracted to 


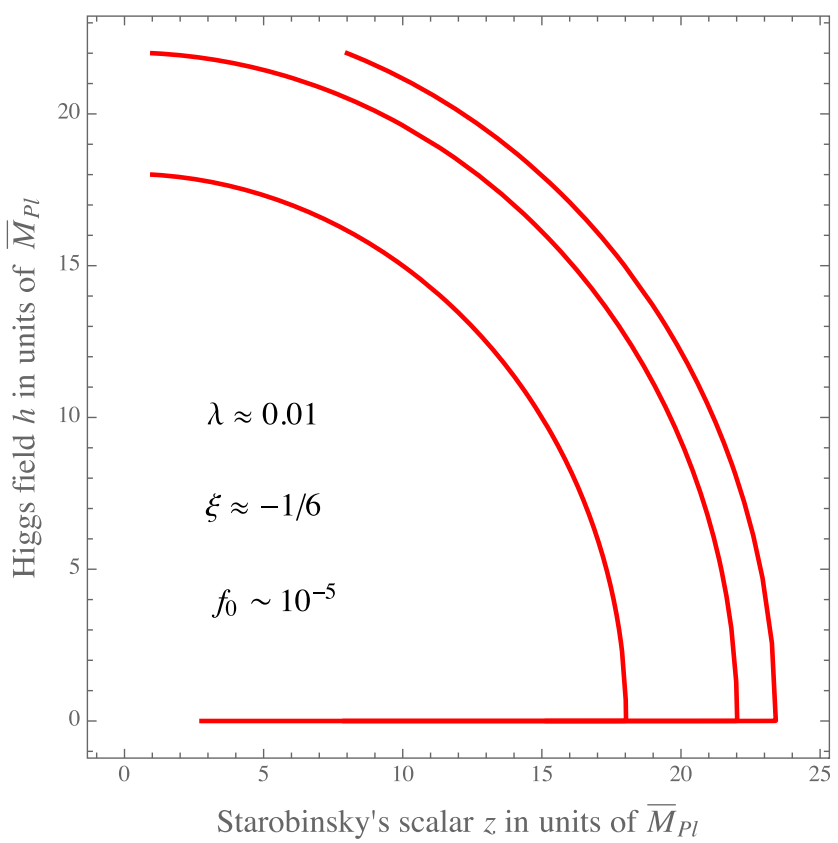

FIG. 1. Inflationary path of the scalar fields.

the line $h=0$ (the running forces $\lambda$ to sizable values, and the potential barrier for $h \neq 0$ is too steep to allow Higgs inflation). Matching the observed $P_{\mathcal{R}}(k)$ leads to $f_{0} \sim 10^{-5}$ and therefore $\xi \approx-1 / 6$ to achieve Higgs mass naturalness, as discussed in Sec. V. Note that inserting $f_{0} \sim 10^{-5}$ in $M_{0}^{\prime}$ leads to $M_{0}^{\prime} \gg m$ as we anticipated in Sec. III.

In Fig. 1, we show the presence of the above-mentioned attractor even for the natural values $\xi \approx-1 / 6$ and $f_{0} \sim 10^{-5}$. It is only when the fields reach the attractor that the slow-roll conditions in (6.13) and (6.16) are satisfied. The curves have been obtained by solving the field equations (6.21) in the slow-roll approximation.

By using Eqs. (6.24) and (6.30), we obtain the predictions for the observable quantities, $n_{s} \approx 0.97$ and $r \approx 0.0031$, for a number of $e$-folds $N \approx 59$. We obtain results that are very close to Starobinsky's predictions $n_{s} \approx$ 0.97 and $r \approx 0.0035$ [10] for the reasons explained above. It is interesting to note that the softened gravity theory under study gives a justification for the $R^{2}$ term in the Lagrangian: as we have previously mentioned, even if we do not introduce it in the classical Lagraingian, quantum corrections generate it.

For negative values of $\lambda$ at the inflationary scales, which are suggested by recent calculations [25,41,42], one should instead require directly that the Higgs is at the EW minimum of the SM potential ${ }^{4}$ large field values of the

\footnotetext{
${ }^{4}$ The quantum and thermal probability of jumping to the true minimum has been recently considered even in the presence of the extra gravitational terms in Eq. (2.2) [43]. The conclusion is that the lifetime of the EW vacuum can well be much bigger than the age of the Universe without tension with any experiment.
}

Higgs above the SM potential barrier would lead to a runaway for the Higgs field, which would not eventually roll toward the EW vacuum.

Therefore, the inflationary nature of the model is close to that of Starobinsky's inflation, in good agreement with current cosmological observations; the differences with Starobinsky's predictions are within current uncertainties, but future observations may give us more information (we will discuss this point in Sec. VII).

\section{CONCLUSIONS AND OUTLOOK}

We have presented a softened gravity theory that, besides having a natural Higgs mass, also possesses a natural QCD $\theta$ angle. Three right-handed neutrinos below the EW scale can explain the neutrino oscillations, DM and BAU and, at the same time, protect the theory from gravitational violation of $C P$ invariance. Contrary to the standard lore, we have shown (within the softened gravity theory studied here) that the smallness of the right-handed Majorana neutrino masses and Yukawa couplings required to explain neutrino oscillations, DM and BAU is not a fine-tuning; their smallness is preserved by the RGEs (which we have determined explicitly), even if the gravity corrections are included. Moreover, low-scale right-handed neutrinos give negligible corrections to the Higgs mass. Therefore, the softened gravity idea is not separate from the idea of lowscale neutrinos; these two ingredients mutually reinforce each other.

The implementation of softened gravity that we have used here presents a spin- 2 heavy ghost in its spectrum. We have shown that this is an unstable state and therefore the basic condition for a Lee-Wick interpretation of this theory is fulfilled. Open problems for the future include the nonperturbative formulation of the theory and the study of its causal structure (which could perhaps be done following the ideas of Refs. [19] and [44], respectively).

Moreover, we have shown that inflation and its predictions are close to those of Starobinsy's $R^{2}$ model. Given the current observational uncertainties, they look actually the same. However, we have found small differences such that they can be distinguished by future observations such as CMBpol [45]. For instance, future sensitivity for $r$ can well be at the level of $10^{-3}$ or below $[45,46]$. We hope that the present work can stimulate future experimental as well as theoretical efforts in distinguishing these theories. A future theoretical goal could be for instance an improved calculation of the inflationary predictions by going beyond the slow-roll approximation.

\section{ACKNOWLEDGMENTS}

We thank A. Strumia, G. Villadoro and M. Shaposhnikov for useful discussions. This work was supported by the 2014 European Research Council (ERC) Grant No. 669668 -NEO-NAT-ERC-AdG-2014. 


\section{APPENDIX A: POLARIZATION TENSORS}

The polarization tensors for a massive spin-2 field are [47]

$$
\begin{gathered}
\text { (in the rest frame) } \quad e_{\mu \nu}^{1}=\frac{1}{\sqrt{2}}\left(\begin{array}{cccc}
0 & 0 & 0 & 0 \\
0 & 1 & 0 & 0 \\
0 & 0 & -1 & 0 \\
0 & 0 & 0 & 0
\end{array}\right), \quad e_{\mu \nu}^{2}=\frac{1}{\sqrt{2}}\left(\begin{array}{ccccc}
0 & 0 & 0 & 0 \\
0 & 0 & 1 & 0 \\
0 & 1 & 0 & 0 \\
0 & 0 & 0 & 0
\end{array}\right), \\
e_{\mu \nu}^{3}=\frac{1}{\sqrt{2}}\left(\begin{array}{cccc}
0 & 0 & 0 & 0 \\
0 & 0 & 0 & 1 \\
0 & 0 & 0 & 0 \\
0 & 1 & 0 & 0
\end{array}\right), \quad e_{\mu \nu}^{4}=\frac{1}{\sqrt{2}}\left(\begin{array}{cccc}
0 & 0 & 0 & 0 \\
0 & 0 & 0 & 0 \\
0 & 0 & 0 & 1 \\
0 & 0 & 1 & 0
\end{array}\right), \quad e_{\mu \nu}^{5}=\frac{\sqrt{2}}{\sqrt{3}}\left(\begin{array}{cccc}
0 & 0 & 0 & 0 \\
0 & 1 / 2 & 0 & 0 \\
0 & 0 & 1 / 2 & 0 \\
0 & 0 & 0 & -1
\end{array}\right) .
\end{gathered}
$$

\section{APPENDIX B: IMAGINARY PART OF LOOP FUNCTIONS}

By using the formula

$$
\frac{1}{a b}=\int_{0}^{1} \frac{d x}{[b+(a-b) x]^{2}},
$$

we find that the function $B_{0}$, which is defined by

$$
B_{0}\left(p^{2}, m_{1}^{2}, m_{2}^{2}\right) \equiv \frac{1}{i \pi^{2}} \int d^{d} q \frac{1}{\left(q^{2}-m_{1}^{2}+i \epsilon\right)\left[(q+p)^{2}-m_{2}^{2}+i \epsilon\right]}
$$

in dimensional regularization (with space-time dimension $d$ ), can be written as

$$
B_{0}\left(p^{2}, m_{1}^{2}, m_{2}^{2}\right)=\frac{1}{i \pi^{2}} \int d^{d} q \int_{0}^{1} d x \frac{1}{\left[q^{2}-m_{1}^{2}+\left(p^{2}+2 q p+m_{1}^{2}-m_{2}^{2}\right) x+i \epsilon\right]^{2}},
$$

and then by introducing the new loop variable $k=q+x p$,

$$
B_{0}\left(p^{2}, m_{1}^{2}, m_{2}^{2}\right) \equiv \frac{1}{i \pi^{2}} \int d^{d} k \int_{0}^{1} d x \frac{1}{\left[k^{2}+x^{2} p^{2}-m_{1}^{2}+\left(p^{2}-2 x p^{2}+m_{1}^{2}-m_{2}^{2}\right) x+i \epsilon\right]^{2}},
$$

and setting $m_{1}=m_{2} \equiv m$,

$B_{0}\left(p^{2}, m^{2}, m^{2}\right) \equiv \frac{1}{i \pi^{2}} \int d^{d} k \int_{0}^{1} d x \frac{1}{\left[k^{2}-F\left(p^{2}, x\right)+i \epsilon\right]^{2}}$,

where

$$
F\left(p^{2}, x\right) \equiv m^{2}+x(x-1) p^{2}
$$

$\operatorname{Im} B_{0}\left(p^{2}, m^{2}, m^{2}\right) \neq 0$ only when $F\left(p^{2}, x\right)$ is negative, that is for $x_{-}<x<x_{+}$where $x_{ \pm}=\left(1 \pm \sqrt{1-4 m^{2} / p^{2}}\right) / 2$, which is possible only for $p^{2}>4 m^{2}$. By performing the Wick rotation, one therefore finds

$$
\begin{aligned}
\operatorname{Im} B_{0}\left(p^{2}, m^{2}, m^{2}\right)= & \frac{1}{\pi^{2}} \int_{x_{-}}^{x_{+}} d x \operatorname{Im} \\
& \times \int d^{4} k_{E} \frac{\theta\left(p^{2}-4 m^{2}\right)}{\left(-k_{E}^{2}+|F|+i \epsilon\right)^{2}},
\end{aligned}
$$

where the label $E$ reminds us that we are now in the Euclidean space (not to be confused with the Einstein frame label). By using spherical coordinates in this space, we obtain

$$
\begin{aligned}
\operatorname{Im} B_{0}\left(p^{2}, m^{2}, m^{2}\right)= & 2 \theta\left(p^{2}-4 m^{2}\right) \int_{x_{-}}^{x_{+}} d x \operatorname{Im} \\
& \times \int_{0}^{\infty} \frac{d r r^{3}}{\left(-r^{2}+|F|+i \epsilon\right)^{2}} .
\end{aligned}
$$

We have 


$$
\begin{aligned}
& \int \frac{d r r^{3}}{\left(-r^{2}+|F|+i \epsilon\right)^{2}} \\
& \quad=\frac{1}{2}\left(\frac{|F|+i \epsilon}{|F|+i \epsilon-r^{2}}+\log \left(|F|+i \epsilon-r^{2}\right)\right) .
\end{aligned}
$$

We can now split the integral $\int_{0}^{\infty} d r$ in the integral $\int_{0}^{\sqrt{|F|+\delta}} d r$ plus $\int_{\sqrt{|F|+\delta}}^{\infty} d r$, where $\delta$ is a positive number, and notice that only the former can give rise to an imaginary part. So

$$
\begin{aligned}
\operatorname{Im} \int_{0}^{\infty} \frac{d r r^{3}}{\left(-r^{2}+|F|+i \epsilon\right)^{2}} & =\frac{1}{2} \operatorname{Im} \log (-\delta+i \epsilon) \\
& =\frac{1}{2} \operatorname{Im} \log (-1)=\frac{\pi}{2} .
\end{aligned}
$$

By inserting this result in (B8), we find

$$
\operatorname{Im} B_{0}\left(p^{2}, m^{2}, m^{2}\right)=\pi \sqrt{1-\frac{4 m^{2}}{p^{2}}} \theta\left(p^{2}-4 m^{2}\right) .
$$

[1] G. F. Giudice, G. Isidori, A. Salvio, and A. Strumia, Softened gravity and the extension of the standard model up to infinite energy, J. High Energy Phys. 02 (2015) 137.

[2] A. Salvio and A. Strumia, Agravity, J. High Energy Phys. 06 (2014) 080.

[3] F. Vissani, Do experiments suggest a hierarchy problem?, Phys. Rev. D 57, 7027 (1998).

[4] M. Farina, D. Pappadopulo, and A. Strumia, A modified naturalness principle and its experimental tests, J. High Energy Phys. 08 (2013) 022.

[5] A. de Gouvea, D. Hernandez, and T. M. P. Tait, Criteria for natural hierarchies, Phys. Rev. D 89, 115005 (2014).

[6] J. D. Clarke and P. Cox, Naturalness made easy: Two-loop naturalness bounds on minimal SM extensions, arXiv: 1607.07446 .

[7] A. A. Starobinsky, A new type of isotropic cosmological models without singularity, Phys. Lett. 91B, 99 (1980).

[8] K. S. Stelle, Classical gravity with higher derivatives, Gen. Relativ. Gravit. 9, 353 (1978).

[9] K. S. Stelle, Renormalization of higher derivative quantum gravity, Phys. Rev. D 16, 953 (1977).

[10] K. Kannike, G. Htsi, L. Pizza, A. Racioppi, M. Raidal, A. Salvio, and A. Strumia, Dynamically induced Planck scale and inflation, J. High Energy Phys. 05 (2015) 065.

[11] A. Salvio and A. Mazumdar, Classical and quantum initial conditions for Higgs inflation, Phys. Lett. B 750, 194 (2015).

[12] F. Capozzi, E. Lisi, A. Marrone, D. Montanino, and A. Palazzo, Neutrino masses and mixings: Status of known and unknown $3 \nu$ parameters, Nucl. Phys. B908, 218 (2016).

[13] S. Dodelson and L. M. Widrow, Sterile-Neutrinos as Dark Matter, Phys. Rev. Lett. 72, 17 (1994).

[14] T. Asaka and M. Shaposhnikov, The nuMSM, dark matter and baryon asymmetry of the universe, Phys. Lett. B 620, 17 (2005).

[15] L. Canetti, M. Drewes, T. Frossard, and M. Shaposhnikov, Dark matter, baryogenesis and neutrino oscillations from right handed neutrinos, Phys. Rev. D 87, 093006 (2013).

[16] E. K. Akhmedov, V. A. Rubakov, and A. Y. Smirnov, Baryogenesis via Neutrino Oscillations, Phys. Rev. Lett. 81, 1359 (1998).
[17] M. Drewes et al., A White Paper on keV Sterile Neutrino Dark Matter, arXiv:1602.04816.

[18] M. Ostrogradski, Mémoire sur les équations différentielles relatives au problème des isopérimètres, Mem. Ac. St. Petersbourg VI, 385 (1850).

[19] A. Salvio and A. Strumia, Quantum mechanics of 4-derivative theories, Eur. Phys. J. C 76, 227 (2016).

[20] T. D. Lee and G. C. Wick, Negative metric and the unitarity of the S matrix, Nucl. Phys. B9, 209 (1969).

[21] I. Antoniadis and E. T. Tomboulis, Gauge invariance and unitarity in higher derivative quantum gravity, Phys. Rev. D 33, 2756 (1986); B. Hasslacher and E. Mottola, Asymptotically free quantum gravity and black holes, Phys. Lett. B 99, 221 (1981).

[22] W. Pauli, On Dirac's new method of field quantization, Rev. Mod. Phys. 15, 175 (1943).

[23] A. Hindawi, B. A. Ovrut, and D. Waldram, Consistent spin two coupling and quadratic gravitation, Phys. Rev. D 53, 5583 (1996).

[24] I. G. Avramidi and A. O. Barvinsky, Asymptotic freedom in higher derivative quantum gravity, Phys. Lett. 159B, 269 (1985).

[25] D. Buttazzo, G. Degrassi, P. P. Giardino, G. F. Giudice, F. Sala, A. Salvio, and A. Strumia, Investigating the near-criticality of the Higgs boson, J. High Energy Phys. 12 (2013) 089.

[26] A. Salvio, A simple motivated completion of the standard model below the Planck scale: Axions and right-handed neutrinos, Phys. Lett. B 743, 428 (2015).

[27] L. H. Chan, T. Hagiwara, and B. A. Ovrut, The effect of heavy particles in low-energy light particle processes, Phys. Rev. D 20, 1982 (1979); S. Randjbar-Daemi, A. Salvio, and M. Shaposhnikov, On the decoupling of heavy modes in Kaluza-Klein theories, Nucl. Phys. B741, 236 (2006); A. Salvio, Ph.D. thesis, International School for Advanced Studies; A. Salvio and M. Shaposhnikov, Chiral asymmetry from a 5D Higgs mechanism, J. High Energy Phys. 11 (2007) 037; J. Elias-Miro, J. R. Espinosa, G. F. Giudice, H. M. Lee, and A. Strumia, Stabilization of the electroweak vacuum by a scalar threshold effect, J. High Energy Phys. 06 (2012) 031. 
[28] M. Fukugita and T. Yanagida, Baryogenesis Without grand unification, Phys. Lett. B 174, 45 (1986).

[29] S. Davidson and A. Ibarra, A lower bound on the righthanded neutrino mass from leptogenesis, Phys. Lett. B 535, 25 (2002).

[30] C. P. Burgess and G. D. Moore, The Standard Model: A Primer (Cambridge University Press, Cambridge, 2006).

[31] R. Delbourgo and A. Salam, The gravitational correction to pcac, Phys. Lett. B 40, 381 (1972).

[32] A. Salvio and A. Mazumdar, Higgs Stability and the $750 \mathrm{GeV}$ diphoton excess, Phys. Lett. B 755, 469 (2016).

[33] T. Chiba and M. Yamaguchi, Extended slow-roll conditions and primordial fluctuations: Multiple scalar fields and generalized gravity, J. Cosmol. Astropart. Phys. 01 (2009) 019.

[34] P. A. R. Ade et al. (Planck Collaboration), Planck 2015 results. XIII. Cosmological parameters, arXiv:1502.01589; Planck 2015 results. XX. Constraints on inflation, arXiv:1502.02114.

[35] Planck Collaboration, Planck 2013 results. XXII. Constraints on inflation, Astron. Astrophys. 571, A22 (2014).

[36] M. Sasaki and E. D. Stewart, A general analytic formula for the spectral index of the density perturbations produced during inflation, Prog. Theor. Phys. 95, 71 (1996).

[37] F. L. Bezrukov and M. Shaposhnikov, The Standard Model Higgs boson as the inflaton, Phys. Lett. B 659, 703 (2008).

[38] F. L. Bezrukov, A. Magnin, and M. Shaposhnikov, Standard Model Higgs boson mass from inflation, Phys. Lett. B 675 , 88 (2009).
[39] F. Bezrukov and M. Shaposhnikov, Standard Model Higgs boson mass from inflation: Two loop analysis, J. High Energy Phys. 07 (2009) 089.

[40] A. Salvio, Higgs inflation at NNLO after the boson discovery, Phys. Lett. B 727, 234 (2013).

[41] G. Degrassi, S. Di Vita, J. Elias-Miro, J. R. Espinosa, G. F. Giudice, G. Isidori, and A. Strumia, Higgs mass and vacuum stability in the Standard Model at NNLO, J. High Energy Phys. 08 (2012) 098.

[42] F. Bezrukov, M. Y. Kalmykov, B. A. Kniehl, and M. Shaposhnikov, Higgs boson mass and new physics, J. High Energy Phys. 10 (2012) 140.

[43] A. Salvio, A. Strumia, N. Tetradis, and A. Urbano, On gravitational and thermal corrections to vacuum decay, J. High Energy Phys. 09 (2016) 054.

[44] B. Grinstein, D. O'Connell, and M. B. Wise, Causality as an emergent macroscopic phenomenon: The Lee-Wick $O(N)$ model, Phys. Rev. D 79, 105019 (2009).

[45] D. Baumann et al. (CMBPol Study Team Collaboration), CMBPol mission concept study: Probing inflation with CMB polarization, AIP Conf. Proc. 1141, 10 (2009).

[46] P. Creminelli, D. L. López Nacir, M. Simonović, G. Trevisan, and M. Zaldarriaga, Detecting primordial $B$-modes after Planck, J. Cosmol. Astropart. Phys. 11 (2015) 031.

[47] H. van Dam and M. J. G. Veltman, Massive and mass-less Yang-Mills and gravitational fields, Nucl. Phys. B22, 397 (1970). 